\title{
ARTICLE \\ Baseline and follow-up activity and functional connectivity in reward neural circuitries in offspring at risk for bipolar disorder
}

\author{
Heather E. Acuff ${ }^{1,2}$, Amelia Versace $\mathbb{i D}^{3}$, Michele A. Bertocci ${ }^{3}$, Cecile D. Ladouceur ${ }^{3}$, Lindsay C. Hanford ${ }^{3}$, Anna Manelis ${ }^{3}$, Kelly Monk ${ }^{3}$ \\ Lisa Bonar ${ }^{3}$, Alicia McCaffrey ${ }^{3}$, Benjamin I. Goldstein ${ }^{4}$, Tina R. Goldstein ${ }^{3}$, Dara Sakolsky $\mathbb{D}^{3}$, David Axelson ${ }^{5}$, Boris Birmaher ${ }^{3}$ and \\ Mary L. Phillips ${ }^{3}$
}

\begin{abstract}
Bipolar disorder (BD) is a serious psychiatric illness with demonstrated abnormalities in reward processing circuitry. Examining this circuitry in youth at familial risk for BD may provide further insight into the underlying mechanisms of BD development. In this study, we compared offspring of bipolar parents (OBP, $n=32$ ), offspring of comparison parents with non-BD psychopathology $(\mathrm{OCP}, n=36)$, and offspring of healthy parents (OHP, $n=39)$ during a functional magnetic resonance imaging reward processing task. Elastic net regression analyses identified 26 activity, functional connectivity (FC), and demographic variables that explained $34.24 \%$ of the variance in group $(\lambda=0.224)$. ANOVA and post-hoc analyses revealed that OBP had significantly lower right ventral striatum-left caudal anterior cingulate $\mathrm{FC}$ to loss (OBP versus OCP: $p=0.028$, OBP versus OHP: $p=0.015$ ) and greater right pars orbitalis-left (OBP versus OCP: $p=0.003$, OBP versus OHP: $p=0.036$ ) and -right (OBP versus OCP: $p=0.001, \mathrm{OBP}$ versus OHP: $p=$ 0.038 ) orbitofrontal cortex FC to reward versus $\mathrm{OCP}$ and $\mathrm{OHP}$, respectively. These findings were not affected by non-BD psychopathology, psychotropic medication use, or symptomatology. There were no changes in, or relationships between, neuroimaging or symptom measures at follow-up (mean(SD) $=2.70(1.22)$ year inter-scan interval) in a subset of youth with followup data (OBP, $n=14 ; \mathrm{OCP}, n=8 ; \mathrm{OHP}, n=19$ ). These findings suggest that lower right ventral striatum-left caudal anterior cingulate FC to loss and greater right pars orbitalis-orbitofrontal cortex FC to reward may be trait-level neural markers that may reflect risk for $\mathrm{BD}$ in at-risk youth. These findings comprise important steps toward identifying neural markers of BD risk, which may enhance early identification and guide interventions for youth at familial risk for BD.
\end{abstract}

Neuropsychopharmacology (2019) 44:1570-1578; https://doi.org/10.1038/s41386-019-0339-2

\section{INTRODUCTION}

Bipolar disorder (BD) is a serious psychiatric illness with a mean prevalence of approximately $2 \%$ in children and adolescents [1]. Its high heritability (59-87\%) places offspring of bipolar parents (OBP) at high risk for developing $\mathrm{BD}$, themselves [2]. One neural circuitry important to $B D$ is reward processing. This has strong associations with impulsive sensation seeking, a personality trait that comprises impulsivity (i.e., prematurely elicited behavior with little/no forethought, reflection, or consideration of the consequences) and sensation seeking (i.e., the inclination/desire to seek/ take risks for new/intense sensations and experiences) [3]. Impulsive sensation seeking is associated with the development of BD [4]. Studies have reported positive associations between impulsive sensation seeking and greater probabilities of adolescents and young adults developing $\operatorname{BD}[5,6]$, as well as mania severity in young adults at risk for BD [7]. Such findings implicate high levels of this trait as potential risk factors for BD [3]. Identifying abnormalities in reward circuitry that are associated with impulsive sensation seeking in youth at risk for BD may elucidate biological markers of specific risk for BD.
While comparing OBP to offspring of healthy parents (OHP) can identify early phenotypes associated with $B D$ risk, these studies have limited abilities to distinguish risk for BD, specifically, from risk for general psychiatric illness, as OBP are also at risk for non$\mathrm{BD}$ psychopathology [8]. Comparing OBP to offspring of comparison parents (OCP) with non-BD psychopathology can distinguish specific $B D$ risk, as OBP is at an approximate seven-fold increased risk for developing BD versus OCP [9]. OCP thus control for risk for non-BD psychiatric disorders and for environmental effects of living with a parent with psychiatric illness. Comparing OBP to both OHP and OCP can provide further insight into underlying mechanisms of $\mathrm{BD}$ development and may lead to enhanced early identification and preventative treatment for youth likely to develop future BD.

Key regions in reward processing circuitry include the ventrolateral prefrontal cortex (vIPFC), important for encoding values of choices and decision-making options [10]; orbitofrontal cortex (OFC), important for encoding reward values and comparing values of different options [11]; ventral striatum (VS), important for anticipation and prediction error [12]; anterior

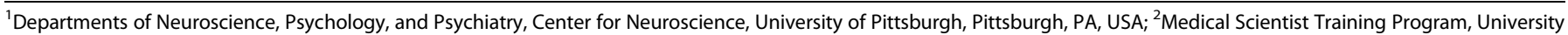

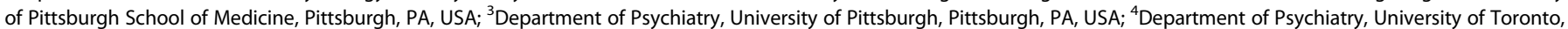
Toronto, ON, Canada and ${ }^{5}$ Department of Psychiatry, Nationwide Children's Hospital and The Ohio State College of Medicine, Columbus, OH, USA Correspondence: Heather E. Acuff (hea19@pitt.edu)

Received: 25 November 2018 Revised: 19 January 2019 Accepted: 6 February 2019

Published online: 13 February 2019 
cingulate cortex (ACC), important for cost-benefit decisionmaking and associating actions with rewards [13]; and amygdala, important for stimulus-value associations [14]. The vIPFC can be further separated into the lateral orbitofrontal cortex [15] and pars orbitalis (Brodmann's Area (BA) 47), pars opercularis (BA44), and pars triangularis (BA45) [16], and the ACC can be separated into rostral (CACC) and caudal (CACC) regions. The $\mathrm{rACC}$ is generally involved in assessing salience of emotional and motivational information and regulating emotional responses, while the CACC is more involved in evaluative and cognitive tasks [17].

Studies have shown abnormalities in reward circuitry in youth and adults with, and at risk for, BD versus healthy controls. Findings in adults with $B D$ include greater right OFC and left vIPFC activity during reward anticipation [18-20]; greater left OFC and amygdala activity during reward reversal [21]; greater [18, 22, 23] and lower [24, 25] VS activity during reward anticipation and receipt; lower ACC activity during reward anticipation [20]; and lower vIPFC-VS functional connectivity (FC) during reward receipt [25]. Findings in youth at risk for BD include greater left lateral OFC activity during reward receipt, lower vIPFC-ACC FC during reward anticipation, and greater vIPFC-ACC FC during loss anticipation [26]. In one study comparing reward circuitry activation in OBP, OCP, and OHP [27], OBP had more inverse bilateral VS-right vIPFC FC versus OCP and OHP during reward and loss receipt [27]. These findings remained after removing youth with non-BD psychiatric disorders and taking psychotropic medications, likely reflecting trait-level neural markers for BD [27].

Our primary goal in this study was to expand upon previous findings by further investigating the differences between OBP, $\mathrm{OCP}$, and $\mathrm{OHP}$ in reward processing circuitry. Our secondary goal was to examine follow-up data in order to help elucidate whether these findings more likely reflected trait- or state-level markers of risk for BD. To achieve these goals, we wished to identify OBPspecific abnormalities in reward processing neural circuitry that were independent of non-BD psychopathology and medications. Elucidating such abnormalities would help identify candidate neural markers of risk for BD versus risk for other psychiatric disorders. As described above, previous findings indicate predominantly greater prefrontal cortical activity, lower ACC activity, abnormal VS activity, and lower FC between prefrontal cortical regions or between the VIPFC and VS in individuals with, and at risk for, $\mathrm{BD}$ relative to OHP and/or OCP, present largely to reward and loss receipt. Previous findings also indicate the consistency of these neural findings when removing youth with psychiatric disorders and medications. We thus hypothesized: (1) OBP would show greater prefrontal cortical activity, lower ACC activity, either greater or lower VS activity, lower prefrontal cortical FC, and lower VIPFC-VS FC to reward and loss receipt versus OCP and OHP; and (2) these findings would be unaffected by non-BD psychopathology and medications. In exploratory analyses, we examined relationships among neuroimaging measures and symptomatology, at baseline and follow-up.

\section{MATERIALS AND METHODS}

Participants

Thirty-two OBP (mean(SD) age =13.95(2.43), 16 females), 36 OCP (mean(SD) age $=14.09(2.32), 14$ females), and 39 OHP (mean(SD) age $=13.90(1.81)$, 18 females) were recruited from the Bipolar Offspring Study [28] and Longitudinal Assessment of Manic Symptoms Study, a parallel study examining neural circuitry functioning in youth with behavioral and emotional dysregulation (Table 1) [29, 30]. Participants were matched for age, sex, IQ, handedness, and highest parental education. Twenty-seven OBP, $25 \mathrm{OCP}$, and 23 OHP were also included in Manelis et al. [27]. Thirteen OBP and 15 OCP had non-BD diagnoses. Six OBP and eight OCP were taking antidepressant, antipsychotic, mood stabilizer, stimulant, and/or non-stimulant medications.

OBP had at least one parent with BD; OCP had at least one parent with a non-BD disorder: MDD, ADHD, and/or an anxiety disorder. Exclusion criteria included history of serious medical illness, head injury, or neurological disorder; $\mathrm{IQ}<70$, assessed with Wechsler Abbreviate Scale of Intelligence [31]; BD, autism, or schizophrenia; MRI contraindication (e.g., pregnancy, metal in the body); substance abuse on the day of the scan or substance abuse disorder in the last 3 months. For OHP, additional exclusion criteria included history of DSM-5 disorder. Before participation, parents and guardians provided written informed consent, and youth provided written informed assent. Participants received monetary compensation.

Psychiatric diagnoses for OBP and OCP were confirmed by a licensed psychiatrist or psychologist before scanning using the Kiddie Schedule for Affective Disorders and Schizophrenia for School-Age Children (K-SADS)-Present and Lifetime Version [32] for offspring and the Structural Clinical Interview for DSM-IV [33] for parents. Symptom assessments included the Screen for Child Anxiety Related Disorders (SCARED) [34, 35], Children's Affective Lability Scale (CALS) [36], Mood and Feelings Questionnaire (MFQ) [37], and K-SADS Depression (KDRS) [32] and Mania (KMRS) [38] Rating Scales to assess four important dimensional predictors of new-onset BD in at-risk youth [39]. Separate parent-reported (-P) and child-reported (-C) SCARED, CALS, and MFQ assessments were administered on the scan day; summary KDRS and KMRS interviews, based on both parent and child information, were administered, on average, 2 months after the scan.

Neuroimaging data acquisition and analyses

Refer to Supplementary Material for task and preprocessing. Participants completed a well-validated card guessing task with reward and loss receipt components (Supplementary Figure 1) [40, 41]. All scan 1 images, and all scan 2 images for OHP, were acquired using a Siemens Magnetom TrimTrio 3T MRI system. All scan 2 images for OBP and OCP were acquired using a Siemens Magnetom Prisma system. Images were preprocessed using Statistical Parametric Mapping (SPM8), including realignment and unwarping steps. First-level fixed effect models were created for activity and FC with trials modeled as epochs with design matrix regressors [42], including 6 directions of motion artifact regressors [43]. Excessive movement (translation $>4 \mathrm{~mm}$ ) were excluded, followed by despiking for remaining participants (http:// afni.nimh.nih.gov/pub/dist/doc/program_help/3dDespike.html). Skull-stripped structural images and associated parcellation and segmentation volumes were resampled into $2 \times 2 \times 2 \mathrm{~mm}^{3} \mathrm{fMRI}$ voxel dimensions before co-registering functional volumes into structural space.

Eighteen regions of interest (ROIs), left and right for each, were anatomically defined using FreeSurfer Center for Morphometric Analysis standard labels: amygdala, caudal (CACC) and rostral (rACC) ACC, OFC (medial orbitofrontal cortex), vIPFC (pars opercularis, pars orbitalis, pars triangularis, and lateral orbitofrontal cortex, separately), and VS (accumbens areas). Task stimulus contrasts included reward versus control and loss versus control, separately. Individual-level averaged BOLD waveforms to the onset of each stimulus type were extracted in native space from anatomic ROls to main stimulus contrasts per task. Generalized psychophysiological interaction analyses assessed task-related connectivity between each seed region and all other aforementioned ROls. Seed regions included bilateral VS, pars opercularis, pars orbitalis, pars triangularis, and lateral orbitofrontal cortex, separately, due to high associations between VS and vIPFC activity and impulsive sensation seeking $[3,22]$. Target regions for each seed included all other aforementioned ROls, separately for left and right regions. 
Table 1. Comparison of OBP, OCP, and OHP

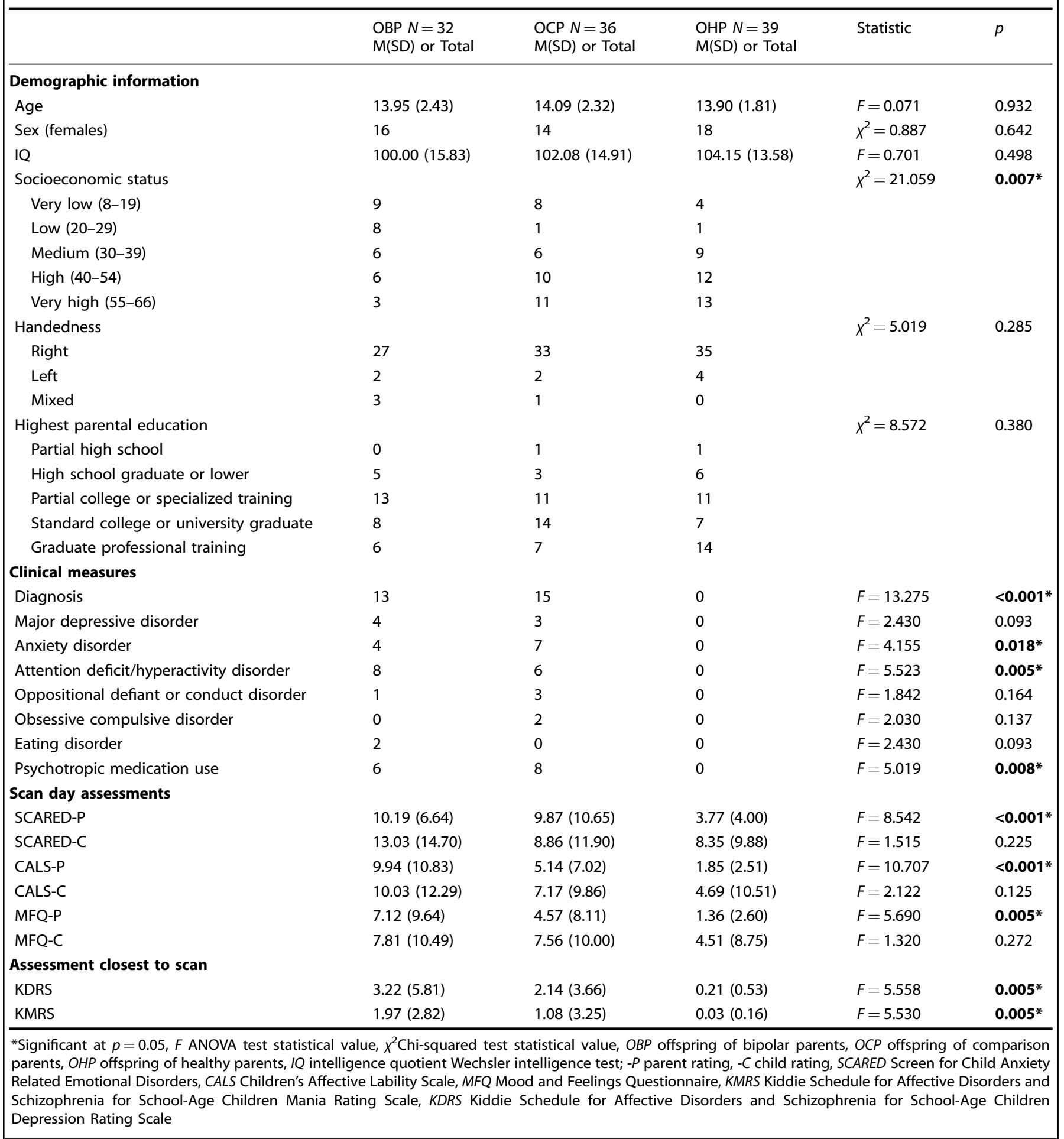

Statistical analyses

A single elastic net regression analysis with $k=10$-fold crossvalidation was used for data selection and reduction [44]. This method was chosen, in part, because it has been shown to be particularly useful when the number of predictor variables is larger than the number of subjects [45]. This model contained two outcome variables: $\mathrm{BD}$ risk (OBP versus $\mathrm{OCP} / \mathrm{OHP}$ ) and general psychiatric disorders risk (OBP/OCP versus OHP). This model also contained 118 predictor variables: demographics (age, sex, IQ, SES (assessed with Hollingshead Four Factor Index of Social Status [46]), handedness, highest parental education), and all possible combinations of activity and FC in all ROls for all contrasts (Supplementary Table 3). Post-hoc pseudo $r$-squared analyses examined the proportion of variance in dependent variables explained by the non-zero predictor variables. Post-hoc ANOVAs and Tukey HSD-corrected tests examined: between-group differences in neuroimaging measures for all non-zero predictors and effects of youth with versus without non-BD disorders. ANOVAs 
and post-hoc $t$-tests examined the effects of medications, and correlation analyses examined the effects of age, on non-zero predictor neuroimaging measures showing significant effects of the group.

ANOVAs and post-hoc $t$-tests examined between-group differences in baseline symptom measures. Correlation analyses examined relationships between neuroimaging and symptom measures, at baseline and follow-up, and relationships between changes in these measures between baseline and follow-up, for all measures showing significant baseline between-group differences. Follow-up analyses occurred in $14 \mathrm{OBP}$, eight $\mathrm{OCP}$, and $19 \mathrm{OHP}$ who had completed second scans (mean(SD) $=2.70(1.22)$ year inter-scan interval). Findings were corrected using Bonferroni corrections.

\section{RESULTS}

Hypothesis Testing 1

Identification of non-zero predictors. Twenty-six of the original 118 non-zero predictor variables optimized model fit using the minimum $\lambda(\lambda=0.224)$ identified by cross-validation (Fig. 1). A pseudo $r$-squared, calculated containing 26 predictors from the model versus an intercept-only model, indicated that $34.24 \%$ of the variance in group was explained by these predictors (Supplementary Table 1). Twenty-two variables were neuroimaging: 2 activity variables to reward (left and right OFC); $10 \mathrm{FC}$ variables to reward (VS-left CACC, VS-right rACC, lateral orbitofrontal cortex-left amygdala, lateral orbitofrontal cortex-left rACC, lateral orbitofrontal cortex-left VS, pars opercularis-right OFC, pars orbitalis-left OFC, pars orbitalis-right CACC, pars orbitalis-right OFC, and pars triangularis-right VS); and $10 \mathrm{FC}$ variables to loss (VS-left CACC, VS-right rACC, lateral orbitofrontal cortex-left amygdala, lateral orbitofrontal cortex-left $\mathrm{rACC}$, pars orbitalis-left rACC, pars orbitalis-right amygdala, pars orbitalis-right CACC, pars triangularis-left rACC, pars triangularis-right amygdala, and pars triangularis-right OFC). Four variables were demographic: IQ, SES (very low and low), and highest parental education (standard college/university graduate).

Between-group differences in neuroimaging predictors. A one-way between-subjects ANOVA examined the effects of group on all non-zero predictors (Fig. 2, Supplementary Table 2). There was a significant effect $(p<0.05)$ of group on VS-left cACC FC to loss $(F(2,104)=4.173, p=0.018)$, pars orbitalis-left $(F(2,104)=7.181$, $p=0.001)$ and -right $(F(2,104)=8.457, p<0.001)$ OFC FC to reward, pars triangularis-right OFC FC to loss $(F(2,104)=3.233$, $p=0.043)$, lateral orbitofrontal cortex-left amygdala FC to reward $(F(2,104)=3.333, p=0.040)$, and SES $(F(2,104)=6.771, p=0.002)$. Post-hoc comparisons (Tukey HSD-corrected) indicated that OBP had significantly lower VS-left CACC FC to loss versus OCP $(p=0.025)$ and $\mathrm{OHP}(p=0.049)$; OBP had greater pars orbitalis-left $(p=0.001)$ and -right $(p<0.001)$ OFC FC to reward versus OCP; OCP had lower pars orbitalis-right OFC FC to reward versus OHP $(p=0.046)$; OBP had greater pars triangularis-right OFC FC to loss versus OCP $(p=0.033)$; and OBP had lower SES versus OCP $(p=0.032)$ and $\mathrm{OHP}(p=0.001)$.

Exploratory effects of left- versus right-sided regions. We additionally explored the lateral nature of the seed regions for the significant FC findings. Two one-way between-subjects ANOVAS (Bonferroni-corrected for 2 tests) and post-hoc $t$-tests (Tukey HSDcorrected) examined between-group differences in VS-left CACC FC to loss, pars orbitalis-left and -right OFC FC to reward, and pars triangularis-right OFC FC to loss using left-sided and right-sided seed regions, separately (Fig. 3). There was a main effect of group on right VS-left CACC FC to loss $(F(2,104)=4.857, p=0.010)$ and right pars orbitalis-left $(F(2,104)=6.140, p=0.003)$ and -right $(F(2,104)=7.552, p=0.001)$ OFC FC to reward. OBP had lower right VS-left CACC FC to loss versus OCP $(p=0.028)$ and OHP ( $p=$ $0.015)$, but greater right pars orbitalis-left $(p=0.003,0.036)$ and -right ( $p=0.001,0.038)$ OFC FC to reward versus OCP and OHP, respectively. There was also a main effect of group on left pars orbitalis-left $(F(2,104)=6.427, p=0.002)$ and -right $(F(2,104)=$ $6.283, p=0.003)$ OFC FC to reward. OBP showed greater FC versus OCP for the left $(p=0.002)$ and right $(p=0.002)$ OFC, respectively. OCP additionally had lower left pars orbitalis-left OFC FC to reward versus OHP $(p=0.045)$.

In summary, OBP significantly differed from both OCP and OHP for three measures: right VS-left CACC FC to loss, right pars orbitalis-left OFC FC to reward, and right pars orbitalis-right OFC FC to reward. These were the focus of all future analyses.

Hypothesis Testing 2

Effects of non-BD disorders. Two one-way between-subjects ANOVAs (Bonferroni-corrected for 2 tests) and post-hoc $t$-tests (Tukey HSD-corrected) examined between-group differences in the three neuroimaging measures that distinguished OBP from both OCP and OHP in youth with versus without non-BD disorders. OBP significantly differed from OCP and OHP only when examining youth without non-BD disorders for the right VS-left cACC $(F(2,76)=4.105, p=0.020)$ and right pars orbitalis-left $(F(2,76)=4.218, p=0.018)$ and -right $(F(2,76)=5.051, p=0.009)$ OFC FC findings. In these youth, OBP had lower right VS-left CACC $(p=0.034,0.033)$ and greater right pars orbitalis-left $(p=0.031$ $0.030)$ and -right ( $p=0.008,0.037)$ OFC FC versus OCP and OHP, respectively. No significant differences were found when comparing OBP and OCP with non-BD disorders.

When medicated youth were excluded and analyses were repeated, main neuroimaging findings remained significant (Supplementary Material). No significant correlations were found between age and any main neuroimaging measures.

\section{Exploratory analyses}

Between-group differences in baseline symptom measures. A oneway between-subjects ANOVA compared the effects of group on 8 baseline symptom measures (Fig. 4). There was a significant effect $(p<0.006$, Bonferroni corrected for 8 tests) of group only on SCARED-P $(F(2,103)=8.542, p<0.001)$, CALS-P $(F(2,103)=10.707$, $p<0.001)$, MFQ-P $(F(2,103)=5.690, p=0.005), \operatorname{KDRS}(F(2,103)=$ $5.558, p=0.005)$, and KMRS $(F(2,103)=5.530, p=0.005)$ scores. Post-hoc comparisons (Tukey HSD-corrected) indicated that OBP had significantly greater CALS-P scores $(p=0.024)$ versus OCP; OBP had greater SCARED-P $(p=0.002)$, CALS-P $(p<0.001)$, MFQ-P $(p=0.003), \operatorname{KDRS}(p=0.004)$, and KMRS $(p=0.004)$ scores versus OHP; and OCP had greater SCARED-P $(p=0.002)$ scores versus OHP.

No significant correlations were found when examining relationships between main neuroimaging (right VS-left CACC, right pars orbitalis-left and -right OFC FC) and symptom (SCARED$P, C A L S-P, M Q-P, K D R S, K M R S$ ) measures that significantly differed among all groups either at baseline, at follow-up, or over time (Supplementary Table 4). Neuroimaging and symptom measures did not significantly differ between first and second scans (Supplementary Table 5).

\section{DISCUSSION}

The goal of this study was to identify neural measures in reward processing circuitry that distinguished OBP from OCP and OHP and determine whether these measures represented trait- or state-level neural markers. Our primary findings indicated that OBP had lower FC between the right VS-left CACC to loss but greater FC between the right pars orbitalis and both the left and right OFC to reward. These findings were not affected by non-BD psychopathology, medications, or symptomatology. 
A Number of Nonzero Coefficients at the Current L1 Norm

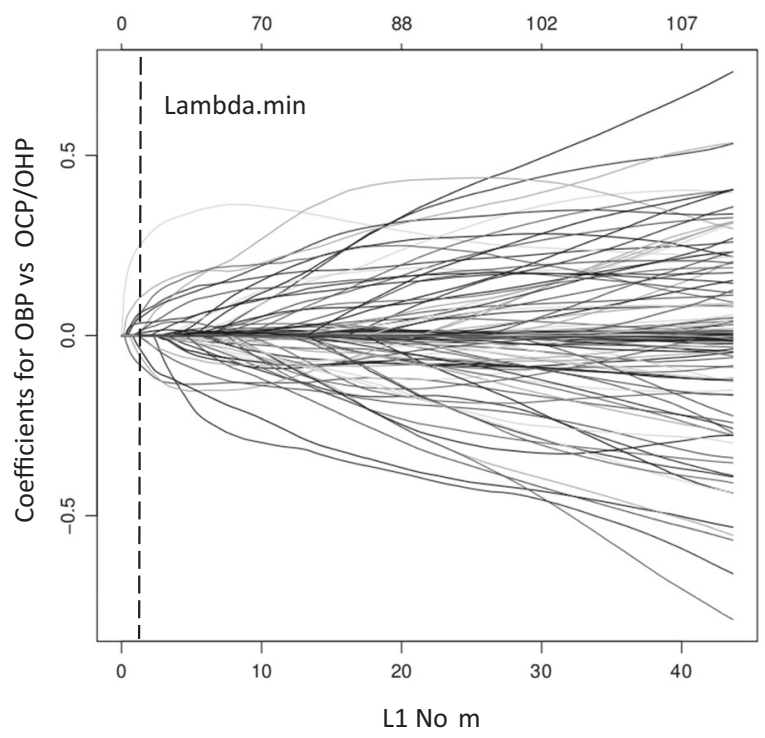

C

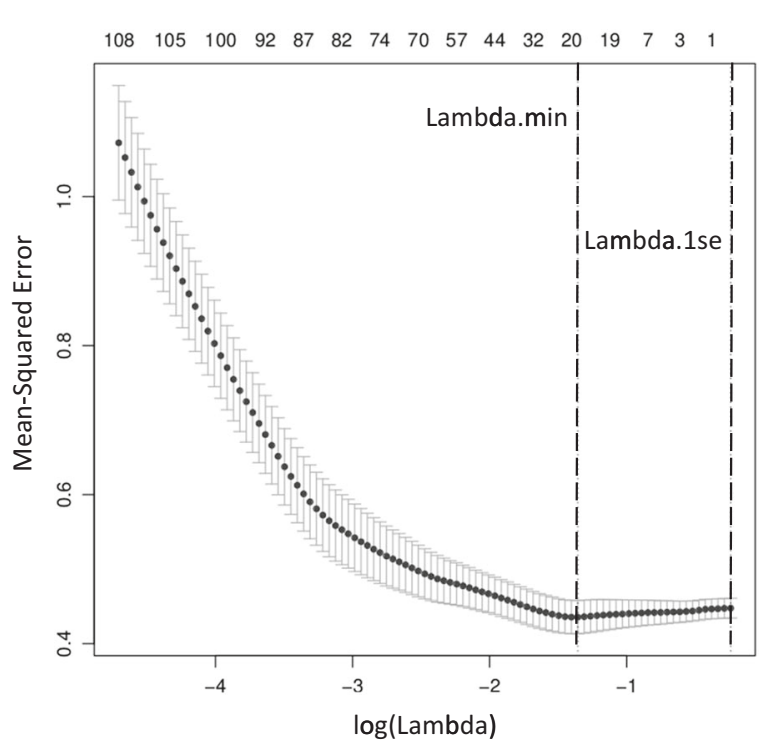

C

Number of Variables
B Number of Nonzero Coefficients at the Current L1 Norm

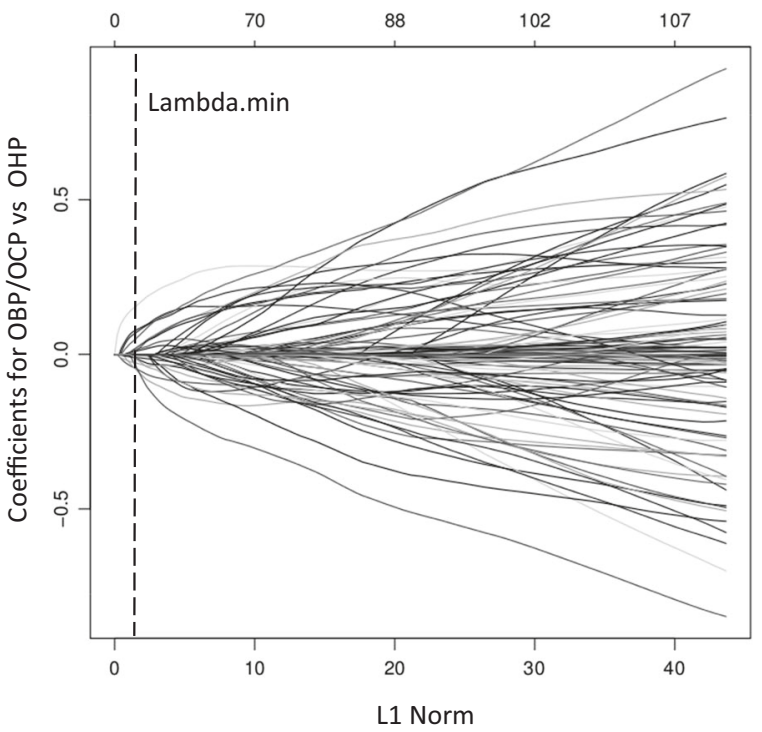




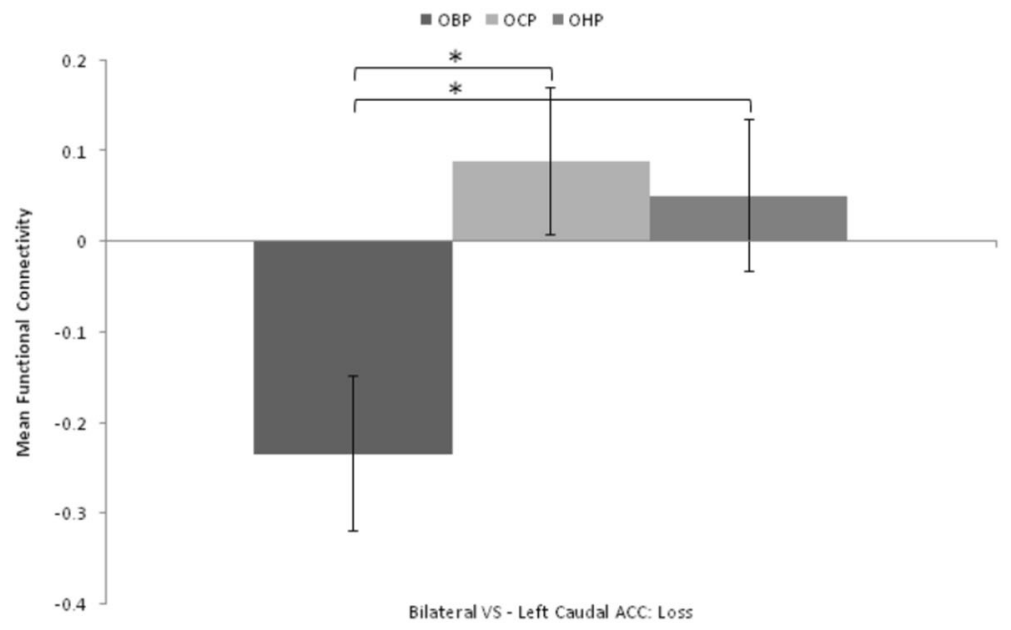

B

\section{Group Differences in vIPFC - OFC FC}

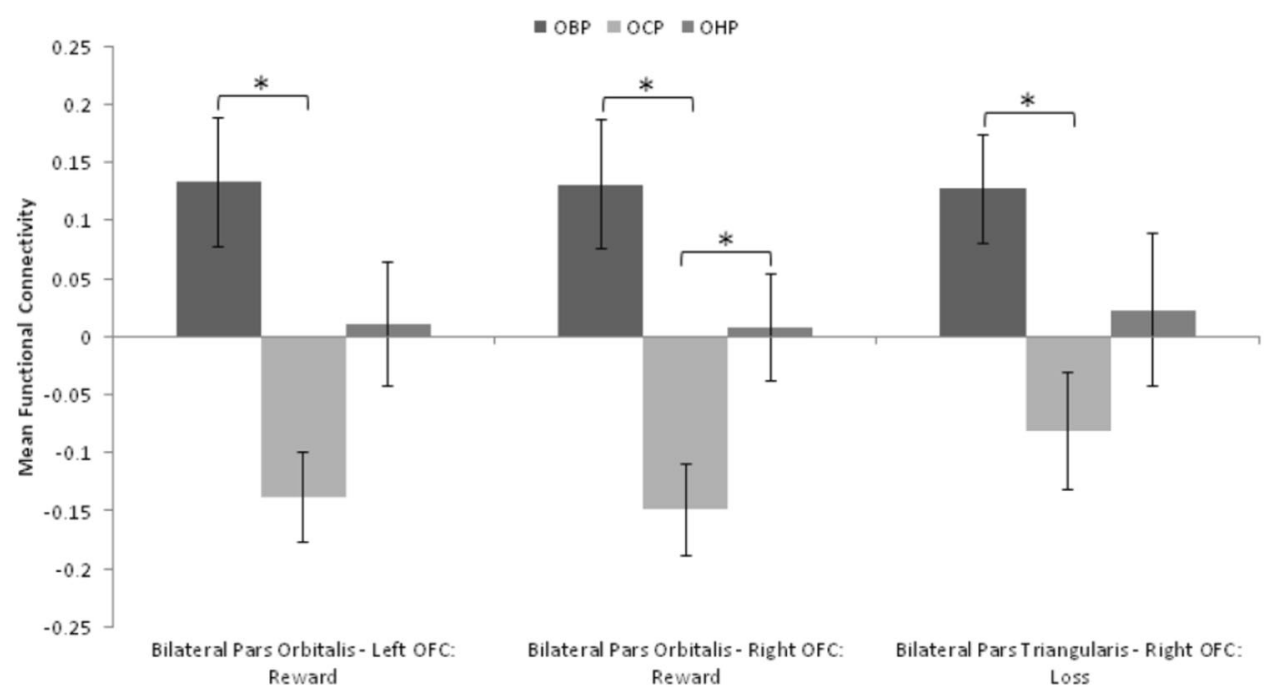

Fig. 2 Group differences in neuroimaging measures. Tukey HSD-corrected group comparisons in significant non-zero predictor neuroimaging measures. a OBP had significantly lower bilateral VS-left caudal ACC functional connectivity when processing loss compared with OCP ( $p=$ $0.025)$ and OHP $(p=0.049)$. b OBP had significantly greater bilateral pars orbitalis-left OFC functional connectivity to reward $(p=0.001)$, bilateral pars orbitalis-right OFC functional connectivity to reward $(p<0.001)$, and bilateral pars triangularis-right OFC functional connectivity to loss $(p=0.033)$ compared with OCP. OCP had significantly lower bilateral pars orbitalis-right OFC functional connectivity to reward compared with OHP $(p=0.046)$. *Significant at $p=0.05$. OBP offspring of bipolar parents, OCP offspring of comparison parents, OHP offspring of healthy parents, VS ventral striatum, ACC anterior cingulate cortex, FC functional connectivity, vIPFC ventrolateral prefrontal cortex, OFC orbitofrontal cortex, SES socioeconomic status

control groups $[18-21,26]$. In line with these findings, we identified greater pars orbitalis-OFC FC to reward in OBP versus both OCP and OHP, likely reflecting greater encoding of, and decision-making about, reward value in OBP. While we found that this relationship distinguished OBP from both groups when examining FC only with the right pars orbitalis, OBP also significantly differed from OCP when examining FC with the left pars orbitalis. The fact that OBP was not significantly distinguished from OHP, as well, might reflect an issue with power. Thus, we may speculate the relationship between the bilateral pars orbitalis and bilateral OFC might be additional neural markers of risk for BP in OBP. Future studies with increased sample sizes are necessary to determine this.

Our findings parallel previous findings that highlighted the importance of the VS and VIPFC in distinguishing OBP from OCP and OHP during reward processing [27]. A previous study, which used standard group-level analyses as opposed to analyses in native space, found that OBP had lower bilateral VS-right vIPFC FC to reward and loss [27], similar to our findings of decreased bilateral (and right) VS-left CACC FC to loss. Additionally, these vIPFC findings were primarily right-sided [27], as in our study. While this previous study showed that the vIPFC had lower connectivity with the VS [27], we showed greater vIPFC-OFC to reward receipt, suggesting a greater encoding of reward values and attunement to reward stimuli in OBP. This highlights the vIPFC as a key region with multiple roles in reward processing circuitry that uniquely distinguish OBP. Additionally, main findings remained significant when excluding youth with non-BD psychopathology and medications, as in previous studies [27]. Furthermore, no relationships were found between main neuroimaging 


\section{A}

\section{Group Differences in Functional Connectivity: Left-Sided Seed}

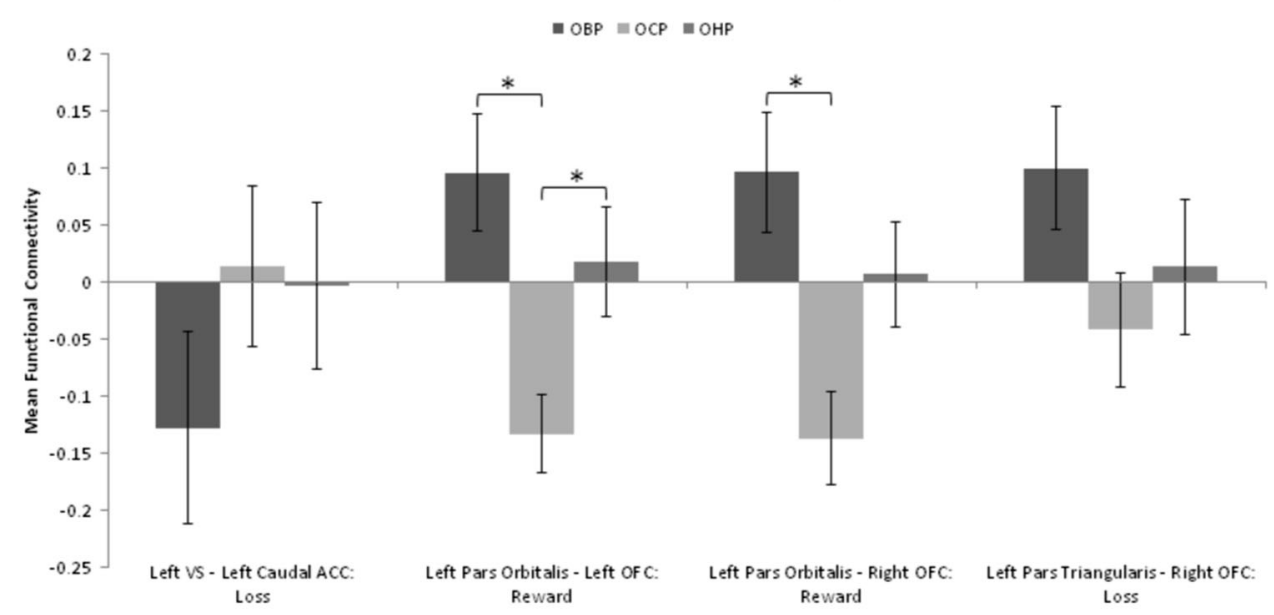

B

\section{Group Differences in Functional Connectivity: Right-Sided Seed}

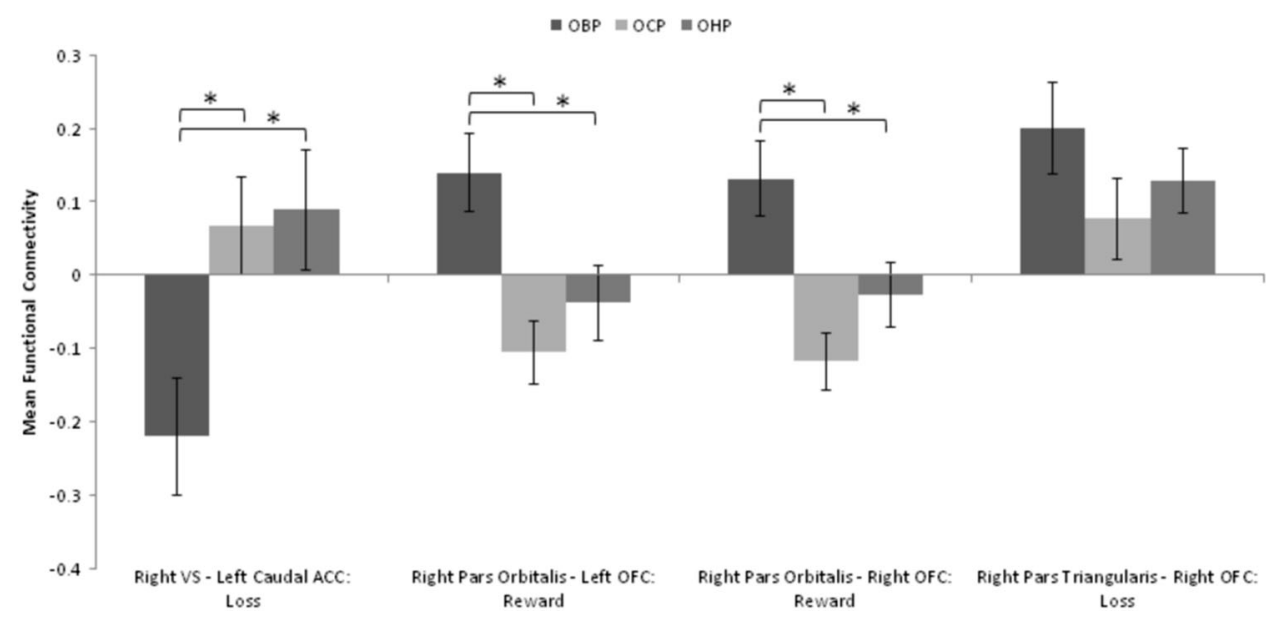

Fig. 3 Group differences in neuroimaging measures: left- versus right-sided seed regions. a Tukey HSD-corrected group comparisons in significant non-zero predictor neuroimaging measures with left-sided seeds. OBP had significantly greater left pars orbitalis-left $(p=0.002)$ and right $(p=0.002)$ OFC functional connectivity to reward compared with OCP. OCP had significantly lower left pars orbitalis-left OFC functional connectivity to reward compared with OHP $(p=0.045)$. b Tukey HSD-corrected group comparisons in significant non-zero predictor neuroimaging measures with right-sided seeds. OBP had significantly lower right VS-left caudal ACC functional connectivity to loss compared with OCP $(p=0.028)$ and OHP $(p=0.015)$. OBP had significantly greater right pars orbitalis-left and right OFC functional connectivity compared with OCP $(p=0.003,0.001)$ and OHP $(p=0.036,0.038)$, respectively. ${ }^{*}$ Significant at $p=0.05$. OBP offspring of bipolar parents, OCP offspring of comparison parents, OHP offspring of healthy parents, VS ventral striatum, ACC anterior cingulate cortex, OFC orbitofrontal cortex

findings and symptomatology, either at baseline or follow-up, and none of these measures showed significant differences in magnitude over time. Together, these findings suggest that lower right VS-left CACC FC to loss and greater right pars orbitalis-OFC FC to reward are more likely to be trait-level neural markers of future $\mathrm{BD}$ risk in $\mathrm{OBP}$, but this will require replication in future studies. This is an important step toward understanding the mechanisms underlying the neural basis of genetic risk for BD.

Additional findings from our study were that OBP had lower SES versus OCP and OHP. Studies have shown that low SES is associated with increased risk for BD [50]. This further suggests that our sample of OBP may be at greater risk for developing BD in the future. An additional finding was that OBP had greater bilateral pars triangularis-right OFC FC to loss versus OCP. This finding did not remain significant when separating the pars triangularis into left and right regions, however. It is possible that this region of the vIPFC has less of a role in reward processing circuitry compared with its other functions, such as verbal semantic retrieval.

This study had limitations. While age did not significantly affect neuroimaging measures, pubertal development cannot be ruled out as a contributing factor in our results. Additionally, recent studies have debated the possible inflation of predictions in neuroimaging studies in individuals with psychiatric disorders [51]; we used a well-validated approach, however, that penalizes complex models using regularization, cross-validation, and sparsity enforcement in model fit. It is possible that the neural markers distinguishing OBP from other groups confer protection against future development of $\mathrm{BD}$ in youth, given that none of the youth in this study had yet developed BD. Only future longitudinal follow-up in these youth will be able to differentiate between risk and protective markers, however.

This is the first study to employ both cross-sectional and longitudinal analyses of reward processing circuitries in youth at 


\section{Group Differences in Symptoms}

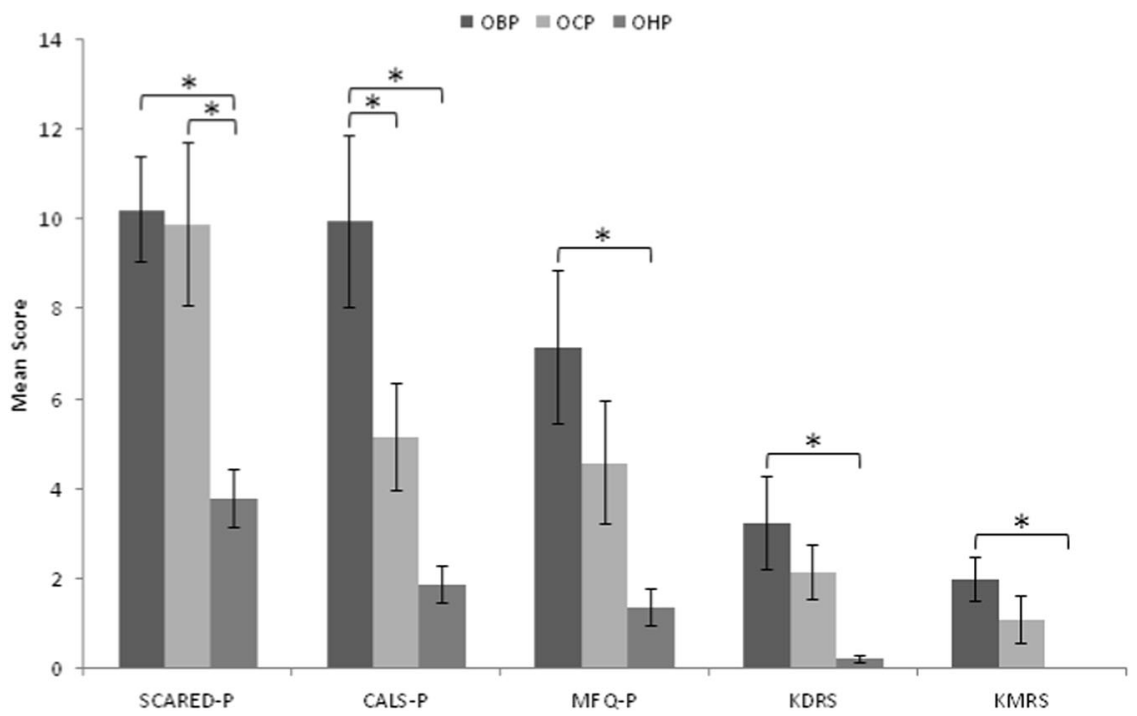

Fig. 4 Group differences in symptom measures. Tukey HSD-corrected group comparisons in symptom measures. Compared with OCP, OBP had significantly greater scores on CALS-P $(p=0.024)$. Compared with OHP, OBP had significantly greater scores on SCARED-P $(p=0.002)$, CALS-P $(p<0.001)$, MFQ-P $(p=0.003)$, KDRS $(p=0.004)$, and KMRS $(p=0.004)$, and OCP had significantly greater scores on SCARED-P $(p=$ 0.002 ). *Significant at $p=0.05$. OBP offspring of bipolar parents, OCP offspring of comparison parents, OHP offspring of healthy parents, SCARED-P Parent-Reported Screen for Child Anxiety Related Disorders, CALS-P Parent-Reported Children's Affective Lability Scale, MFQ ParentReported Mood and Feelings Questionnaire, KDRS Kiddie Schedule for Affective Disorders and Schizophrenia for School-Age Children Depression Rating Scale, KMRS Kiddie Schedule for Affective Disorders and Schizophrenia for School-Age Children Mania Rating Scale

risk for BD versus comparative at-risk and healthy control groups. We show that lower right VS-left CACC FC to loss and greater right pars orbitalis-OFC FC to reward significantly distinguish OBP from both OCP and OHP. These findings are independent of non-BD psychopathology, medication use, and symptomatology and do not significantly change at follow-up, rendering them more likely trait- than state-level neural markers, and which may reflect either risk for, or protection against, BD in at-risk youth. Our findings comprise an important step toward identifying neural markers of $\mathrm{BD}$ risk to aid in enhanced early identification, and guide interventions, for BD at-risk youth.

\section{FUNDING AND DISCLOSURE}

This work was supported by the National Institute of Mental Health (BB and MLP, grant number R01 MH060952-16; HEA, grant number F30 MH111102-01A1). Dr. Acuff, Dr. Versace, Dr. Bertocci, Dr. Hanford, Dr. Ladouceur, Dr. Manelis, Dr. Monk, Dr. Bonar, Dr. McCaffrey, Dr. Goldstein, and Dr. Phillips have no financial interests or potential conflicts of interest. Dr. Axelson has served as a consultant for Janssen Research and received royalties from UpToDate. Dr. Tina Goldstein receives research funding from NIMH, AFSP, and the Brain and Behavior Foundation and receives royalties from Guilford Press. Dr. Sakolsky serves as an editorial board member of Child \& Adolescent Psychopharmacology News and specialty consultant for the Prescriber's Letter. She has received a consultant fee of $\$ 300$ from L.E.K. Consulting in 2015. She currently receives research funding from NIMH and has received funding from NARSAD, the World's Leading Charity Dedicated to Mental Health Research. Dr. Birmaher receives or will receive royalties for publications from Random House, Inc. (New Hope for Children and Teens with Bipolar Disorder), Lippincott Williams \& Wilkins (Treating Child and Adolescent Depression), and UpToDate. He is employed by the University of Pittsburgh and the University of Pittsburgh Medical Center/Western Psychiatric Institute and Clinic and receives research funding from NIMH.

\section{ACKNOWLEDGEMENTS}

The authors would like to acknowledge the participants and their families for their contributions to this study. The authors would also like to acknowledge the individuals involved in The Longitudinal Assessment of Manic Symptoms (LAMS) Study: Department of Psychiatry, Ohio State University, Columbus, OH: L. Eugene Arnold, Mary A. Fristad. Department of Psychiatry, Western Psychiatric Institute and Clinic, University of Pittsburgh Medical Center, University of Pittsburgh, Pittsburgh, PA: Genna Bebko, Mary Kay Gill, Claudiu Schirda, Michael Travis. Department of Psychiatry and Behavioral Neuroscience, Wayne State University, Detroit, MI: Vaibhav A. Diwadkar. Department of Psychiatry, Johns Hopkins University, Baltimore, MD: Robert L. Findling. Department of Radiology, University Hospitals Case Medical Center/Case Western Reserve University, Cleveland, OH: Scott K. Holland. Department of Child and Adolescent Psychiatry, New York University School of Medicine, New York City, NY: Sarah M. Horwitz. Research Institute at Nationwide Children's Hospital, Columbus, OH: Robert A. Kowatch. University Hospitals Case Medical Center/Case Western Reserve University, Cleveland, OH: Jeffrey L. Sunshine. Department of Psychology, University of North Carolina at Chapel Hill, Chapel Hill, NC: Eric A. Youngstrom.

\section{ADDITIONAL INFORMATION}

Supplementary Information accompanies this paper at (https://doi.org/10.1038/ s41386-019-0339-2).

Publisher's note: Springer Nature remains neutral with regard to jurisdictional claims in published maps and institutional affiliations.

\section{REFERENCES}

1. Van Meter AR, Moreira AL, Youngstrom EA. Meta-analysis of epidemiologic studies of pediatric bipolar disorder. J Clin Psychiatry. 2011;72:1250-6.

2. Smoller JW, Finn CT. Family, twin, and adoption studies of bipolar disorder. Am J Med Genet Part C: Semin Med Genet. 2003;123:48-58.

3. Chase HW, Fournier JC, Bertocci MA, Greenberg T, Aslam H, Stiffler R, et al. A pathway linking reward circuitry, impulsive sensation-seeking and risky decisionmaking in young adults: identifying neural markers for new interventions. Transl Psychiatry. 2017;7:e1096.

4. Urošević S, Abramson LY, Harmon-Jones E, Alloy LB. Dysregulation of the behavioral approach system (BAS) in bipolar spectrum disorders: review of theory and evidence. Clin Psychol Rev. 2008;28:1188-205. 
5. Meyer B, Johnson SL, Carver CS. Exploring behavioral activation and inhibition sensitivities among college students at risk for bipolar spectrum symptomatology. J Psychopathol Behav Assess. 1999;21:275-92.

6. Giovanelli A, Hoerger M, Johnson SL, Gruber J. Impulsive responses to positive mood and reward are related to mania risk. Cogn Emot. 2013;27:1091-104.

7. Alloy LB, Bender RE, Whitehouse WG, Wagner CA, Liu RT, Grant DA, et al. High behavioral approach system (BAS) sensitivity, reward responsiveness, and goalstriving predict first onset of bipolar spectrum disorders: A prospective behavioral high-risk design. J Abnorm Psychol. 2012;121:339.

8. Chang KD, Steiner H, Ketter TA. Psychiatric phenomenology of child and adolescent bipolar offspring. J Am Acad Child Adolesc Psychiatry. 2000;39:453-60.

9. Axelson D, Goldstein B, Goldstein T, Monk K, Yu H, Hickey MB, et al. Diagnostic precursors to bipolar disorder in offspring of parents with bipolar disorder: a longitudinal study. Am J Psychiatry. 2015;172:638-46.

10. Walton ME, Behrens TE, Noonan MP, Rushworth MF. Giving credit where credit is due: orbitofrontal cortex and valuation in an uncertain world. Ann N Y Acad Sci. 2011;1239:14-24.

11. Boorman ED, Behrens TE, Woolrich MW, Rushworth MF. How green is the grass on the other side? Frontopolar cortex and the evidence in favor of alternative courses of action. Neuron. 2009;62:733-43.

12. Schultz W, Tremblay L, Hollerman JR. Reward processing in primate orbitofrontal cortex and basal ganglia. Cereb Cortex. 2000;10:272-83.

13. Rushworth MF, Noonan MP, Boorman ED, Walton ME, Behrens TE. Frontal cortex and reward-guided learning and decision-making. Neuron. 2011;70:1054-69.

14. Baxter MG, Murray EA. The amygdala and reward. Nat Rev Neurosci. 2002;3:563.

15. Hooker, C. I. \& Knight, R. T. The orbitofrontal cortex in the inhibitory control of emotion. Vol. Chapter 11 (2006).

16. Badre $D$, Wagner AD. Left ventrolateral prefrontal cortex and the cognitive control of memory. Neuropsychologia. 2007;45:2883-901.

17. Bush G, Luu P, Posner MI. Cognitive and emotional influences in anterior cingulate cortex. Trends Cogn Sci. 2000;4:215-22.

18. Nusslock R, Almeida JR, Forbes EE, Versace A, Frank E, LaBarbara EJ, et al. Waiting to win: elevated striatal and orbitofrontal cortical activity during reward anticipation in euthymic bipolar disorder adults. Bipolar Disord. 2012;14:249-60.

19. Bermpohl F, Kahnt T, Dalanay U, Hagele C, Sajonz B, Wegner T, et al. Altered representation of expected value in the orbitofrontal cortex in mania. Human brain Mapp. 2010;31:958-69.

20. Chase HW, Nusslock R, Almeida JR, Forbes EE, LaBarbara EJ, Phillips ML, et al. Dissociable patterns of abnormal frontal cortical activation during anticipation of an uncertain reward or loss in bipolar versus major depression. Bipolar Disord. 2013;15:839-54.

21. Linke J, King AV, Rietschel M, Strohmaier J, Hennerici M, Gass A, et al. Increased medial orbitofrontal and amygdala activation: evidence for a systems-level endophenotype of bipolar I disorder. Am J Psychiatry. 2012;169:316-25.

22. Caseras X, Lawrence NS, Murphy K, Wise RG, Phillips ML. Ventral striatum activity in response to reward: differences between bipolar I and II disorders. Am J Psychiatry. 2013;170:533-41.

23. Phillips ML, Kupfer DJ. Bipolar disorder diagnosis: challenges and future directions. Lancet. 2013;381:1663-71.

24. Abler B, Greenhouse I, Ongur D, Walter H, Heckers S. Abnormal reward system activation in mania. Neuropsychopharmacology. 2008;33:2217.

25. Trost S, Diekhof EK, Zvonik K, Lewandowski M, Usher J, Keil M, et al. Disturbed anterior prefrontal control of the mesolimbic reward system and increased impulsivity in bipolar disorder. Neuropsychopharmacology. 2014;39:1914.

26. Singh MK, Kelley RG, Howe ME, Reiss AL, Gotlib IH, Chang KD, et al. Reward processing in healthy offspring of parents with bipolar disorder. JAMA Psychiatry. 2014;71:1148-56.

27. Manelis A, Ladouceur CD, Graur S, Monk K, Bonar LK, Hickey MB, et al. Altered functioning of reward circuitry in youth offspring of parents with bipolar disorder. Psychol Med. 2016;46:197-208.

28. Birmaher B, Axelson D, Monk K, Kalas C, Goldstein B, Hickey MB, et al. Lifetime psychiatric disorders in school-aged offspring of parents with bipolar disorder: the Pittsburgh Bipolar Offspring study. Arch General Psychiatry. 2009;66:287-96.

29. Findling RL, Youngstrom EA, Fristad MA, Birmaher B, Kowatch RA, Arnold LE, et al. Characteristics of children with elevated symptoms of mania: the
Longitudinal Assessment of Manic Symptoms (LAMS) study. J Clin Psychiatry. 2010;71:1664.

30. Horwitz SM, Demeter CA, Pagano ME, Youngstrom EA, Fristad MA, Arnold LE, et al. Longitudinal Assessment of Manic Symptoms (LAMS) study: background, design and initial screening results. J Clin Psychiatry. 2010;71:1511.

31. Wechsler D. Wechsler Abbreviated Scale of Intelligence. San Antonio, TX: The Psychological Corporation; 1999.

32. Kaufman J, Birmaher B, Brent D, Rao U, Flynn C, Moreci $P$, et al. Schedule for affective disorders and schizophrenia for school-age children-present and lifetime version (K-SADS-PL): initial reliability and validity data. J Am Acad Child Adolesc Psychiatry. 1997;36:980-8.

33. First MB, Spitzer RL, Gibbon M, Williams J. Structured Clinical Interview for DSM-IV Axis I Disorders, Clinician Version (SCID-CV). Washington, DC: American Psychiatric Press; 1996.

34. Birmaher B, Khetarpal S, Brent D, Cully M, Balach L, Kaufman J, et al. The screen for child anxiety related emotional disorders (SCARED): scale construction and psychometric characteristics. J Am Acad Child Adolesc Psychiatry. 1997;36:545-53.

35. Birmaher B, Brent DA, Chiappetta L, Bridge J, Monga S, Baugher M, et al. Psychometric properties of the Screen for Child Anxiety Related Emotional Disorders (SCARED): a replication study. J Am Acad Child Adolesc Psychiatry. 1999;38:1230-6.

36. Gerson AC, Gerring JP, Freund L, Joshi PT, Capozzoli J, Brady K, et al. The Children's Affective Lability Scale: a psychometric evaluation of reliability. Psychiatry Res. 1996;65:189-98.

37. Sund A, Larsson B, Wichstrøm L. Depressive symptoms among young Norwegian adolescents as measured by the Mood and Feelings Questionnaire (MFQ). Eur Child Adolesc Psychiatry. 2001;10:222-9.

38. Axelson D, Birmaher B, Brent D, Wassick S, Hoover C, Bridge J, et al. A preliminary study of the Kiddie Schedule for Affective Disorders and Schizophrenia for School-Age Children mania rating scale for children and adolescents. J Child Adolesc Psychopharmacol. 2003;13:463-470.

39. Hafeman DM, Merranko J, Axelson D, Goldstein BI, Goldstein T, Monk K, et al. Toward the definition of a bipolar prodrome: dimensional predictors of bipolar spectrum disorders in at-risk youths. Am J Psychiatry. 2016;173:695-704.

40. Bebko G, Bertocci MA, Fournier JC, Hinze AK, Bonar L, Almeida JR, et al. Parsing dimensional vs diagnostic category-related patterns of reward circuitry function in behaviorally and emotionally dysregulated youth in the longitudinal assessment of manic symptoms study. JAMA Psychiatry. 2014;71:71-80.

41. Forbes EE, Hariri AR, Martin SL, Silk JS, Moyles DL, Fisher PM, et al. Altered striatal activation predicting real-world positive affect in adolescent major depressive disorder. Am J Psychiatry. 2009;166:64-73.

42. Ashburner J, Friston K. Unified segmentation. Neurolmage. 2005;26:839-51.

43. Satterthwaite TD, Elliott MA, Gerraty RT, Ruparel K, Loughead J, Calkins ME, et al. An improved framework for confound regression and filtering for control of motion artifact in the preprocessing of resting-state functional connectivity data. Neurolmage. 2013;64:240-56.

44. Friedman J, Hastie T, Somin N, Tibshirani R. GLMNET. 2.0-2 edn. 2014.

45. Zou H, Hastie T. Regularization and variable selection via the elastic net. J R Stat Soc: Ser B (Stat Methodol). 2005;67:301-20.

46. Hollingshead AB. Four-factor index of social status. New Haven, Connecticut: Yale University Department of Sociology; 1975.

47. Jung YC, Schulte T, Muller-Oehring EM, Hawkes W, Namkoong K, Pfefferbaum A, et al. Synchrony of anterior cingulate cortex and insular-striatal activation predicts ambiguity aversion in individuals with low impulsivity. Cereb Cortex. 2013;24:1397-408.

48. van Holst RJ, Chase HW, Clark L. Striatal connectivity changes following gambling wins and near-misses: associations with gambling severity. Neurolmage Clin. 2014;5:232-9.

49. Snow PJ. The structural and functional organization of cognition. Front Human Neurosci. 2016;10:501.

50. Kessler RC, Rubinow D, Holmes C, Abelson J, Zhao S. The epidemiology of DSMIII-R bipolar I disorder in a general population survey. Psychol Med. 1997;27:1079-89.

51. Whelan $\mathrm{R}$, Garavan $\mathrm{H}$. When optimism hurts: inflated predictions in psychiatric neuroimaging. Biol Psychiatry. 2014;75:746-8. 